\title{
Efficacy and safety of endoscopic submucosal dissection for superficial cancer of the cervical esophagus
}

\section{다 (1) $\odot$}

Authors

Toshiro lizuka ${ }^{1,2}$, Daisuke Kikuchi ${ }^{1}$, Shu Hoteya ${ }^{1}$, Yoshiaki Kajiyama ${ }^{2}$, Mitsuru Kaise ${ }^{1}$

Institutions

1 Department of Gastroenterology, Toranomon Hospital, Tokyo, Japan

2 Department of Upper Gastrointestinal Surgery, Juntendo University, Tokyo, Japan

submitted 4.4.2017

accepted after revision 2.5.2017

\author{
Bibliography \\ DOI https://doi.org/10.1055/s-0043-112493 | \\ Endoscopy International Open 2017; 05: E736-E741 \\ (c) Georg Thieme Verlag KG Stuttgart · New York \\ ISSN 2364-3722
}

Corresponding author

Toshiro lizuka, MD, Department of Gastroenterology,

Toranomon Hospital , 2-2-2 Toranomon, Minato-ku, Tokyo

105-8470, Japan

Fax: +81-3-3582-7068

t-iizuka@toranomon.gr.jp

\section{ABSTRACT}

Background and study aims Cervical esophageal cancer (CEC) is a less common form of cancer and often locally advanced at the time of diagnosis; thus, survival rates for pa- tients with CEC remain poor. However, no reports exist on results of endoscopic submucosal dissection (ESD) for superficial cancer at the cervical esophagus. The aim of this retrospective study was to elucidate the clinicopathological features and clinical outcomes of ESD for superficial CEC. Patients and methods ESD was performed on 891 lesions (in 662 patients) for superficial esophageal cancer from January 2008 to December 2015. Of these, 45 lesions (45 patients) were enrolled in the case group (CEC), and 405 lesions (375 patients) were enrolled in the control group (superficial cancer in the middle thoracic esophagus). The safety of ESD, including $R 0$ resection rate and adverse events, and the efficacy, such as the local recurrence rate and overall survival rate, were evaluated.

Results The R0 resection rate was $91.1 \%$ in the case group and $96 \%$ in the control group. The rate of esophageal stricture was significantly higher in the case group (20\%) than in the control group (6.6\%). There was no local recurrence, and the 3 -year survival rate was $88.4 \%$ in the case group and $96.7 \%$ in the control group.

Conclusions ESD for superficial cancer in the cervical esophagus was achieved safely, and successful local control was also confirmed. However, the esophageal stricture after ESD was more frequent.

\section{Introduction}

Cervical esophageal cancer (CEC) is a less common form of cancer that comprises less than $2 \%$ to $10 \%$ of all esophageal cancer [1]. The cervical esophageal region is generally contracted because of the upper sphincter muscle. Therefore, it is difficult to observe this area, and it is challenging to detect CEC at an early stage. CECs are often locally advanced at the time of diagnosis, infiltrating nearby anatomical structures including the cricoid or thyroid gland. Moreover, patients with CEC often present with lymph node metastases [2]. Thus, historically, surgery has been the standard treatment for CEC. A 1994 review found that the 5 -year survival rate with surgery was $12 \%$ to $27 \%$ and that surgical resection was associated with a postoperative mortality rate of $6 \%$ to $20 \%$ and significant morbidity [3]. Over the last decades, new surgical strategies, such as minimally invasive surgery and neoadjuvant (chemo)radiotherapy, have developed [4]. However, surgical treatment still has a great risk of major complications and high morbidity and mortality rates $[5,6]$. Finally, survival rates of patients with CEC remain poor [7].

Endoscopic instrumentation has been progressing, and magnified endoscopy with narrow band imaging (NBI) or a scope attachment is available for screening. The number of superficial cancers in the cervical esophagus is expected to increase. Endoscopic submucosal dissection (ESD) was applied for superficial esophageal cancer, and it has widespread use as the standard treatment for superficial cancer of the upper and lower gastrointestinal tract. However, there is no report about the results of ESD for superficial CEC. 

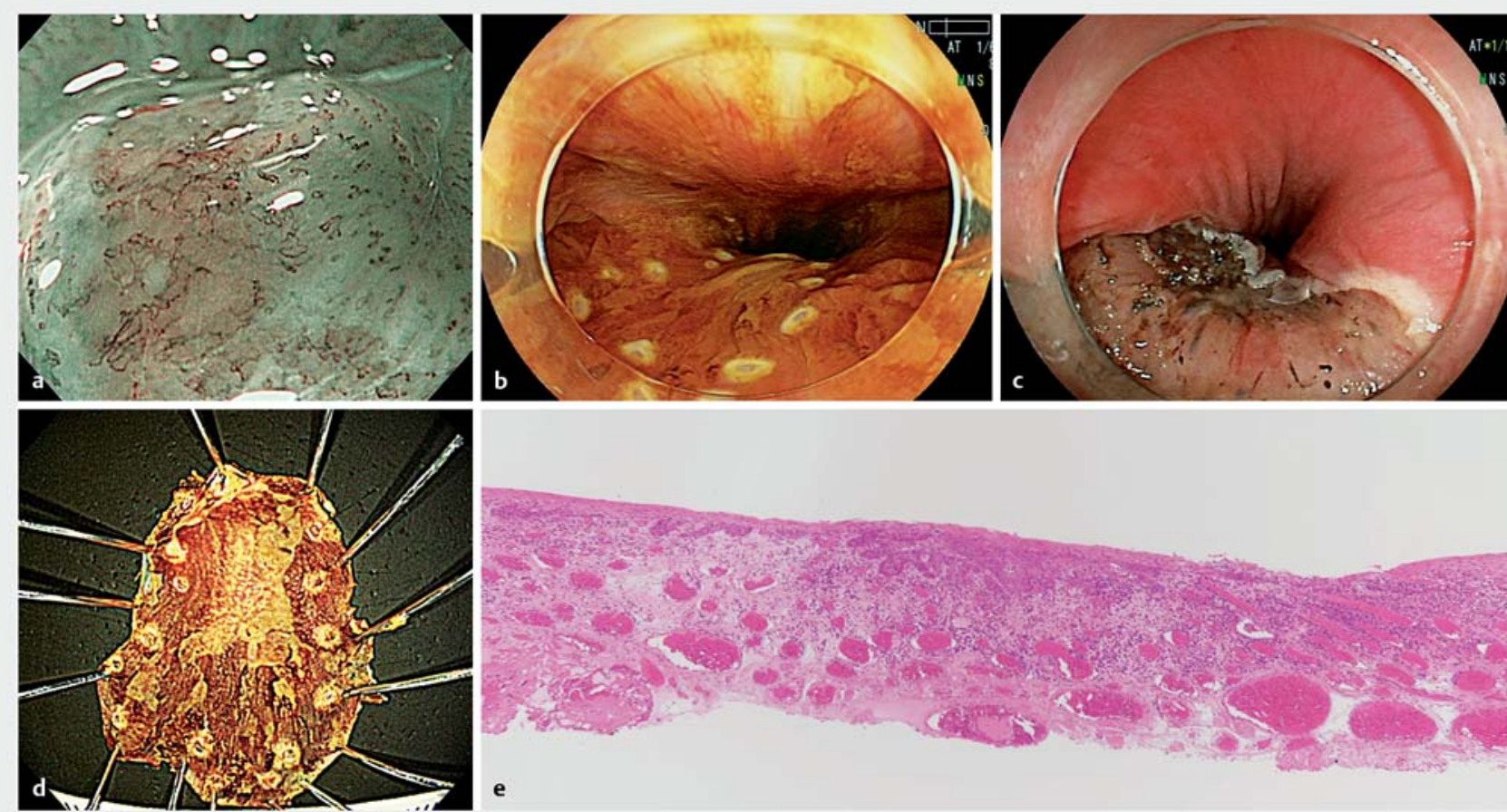

- Fig. 1 ESD for superficial cancer in the cervical esophagus. a The lesion was located at the cervical esophagus, and dilated irregular microvessels can be seen by magnified endoscopy with NBI. b Marking dots were made around the lesion after spraying isodine under general anesthesia. c Submucosal dissection was conducted, and the lesion was removed en bloc. $\mathbf{d}$ The resected specimen was examined histologically. e Histopathological examination showed the SCC invaded into lamina propria mucosae without lymphovascular involvement.

Therefore, we performed a retrospective evaluation of a series of consecutive patients in a single center, who underwent ESD for superficial CEC. The aim of this study was to elucidate the clinicopathological features and clinical outcomes of ESD for superficial CEC compared with those for superficial cancer in the mid-thoracic esophagus.

\section{Patients and methods}

\section{Case group patients}

A total of 662 patients with 891 lesions underwent ESD for esophageal cancers from January 2008 to December 2015 in Toranomon Hospital. Among them, patients who met the following criteria were enrolled as the case group. First, the lesion was located at the cervical esophagus. Cervical esophagus was defined as the esophagus from the esophageal entrance to $20 \mathrm{~cm}$ from the teeth endoscopically. Second, squamous cell carcinoma was proved histologically. Exclusion criteria were as follows: a lesion with an entire circumferential mucosal defect, a recurrent lesion after radiotherapy, and a lesion located near scar tissue. A total of 45 patients were enrolled as the case group.

\section{Control group patients}

Patients who had esophageal squamous cell carcinoma in the middle thoracic esophagus were enrolled as the control group. They all received ESD during the same period as the case group, and they met the same exclusion criteria. A total of 375 patients with 405 lesions were enrolled as the control group.

\section{ESD procedure}

ESD was performed by all of our staff, including non-experts (endoscopists with less than 10 years' experience performing esophageal ESD or experience of less than 100 cases) ( $>$ Fig.1). Either general anesthesia or consciousness anesthesia was chosen by the surgeon. For ESD, a single-channel upper gastrointestinal endoscope with a water-jet system (EG450RD5; Fujifilm, Tokyo, Japan or GIF-Q260 J; Olympus Medical Systems, Tokyo, Japan) was used with a transparent cap attached to the endoscope tip. Flex knife or Dual knife (Olympus Medical Systems, Tokyo, Japan) and a standard electrosurgical generator (ICC 200 or VIO300D; ERBE Elektromedizin $\mathrm{GmbH}$, Tübingen, Germany) were used during ESD. After isodine spraying and delineating the tumor margin, marking dots were placed outside the margins of the tumor using the Flex or Dual knife. Marking dots were completed when the tip of the knife was attached to the mucosa and high-frequency current flowed. First, the submucosal layer was injected locally and an incision was made from the distal side of the lesion. The injection solution consisted of $10 \%$ glycerin and a small amount of indigo carmine and epinephrine. Then, the proximal mucosa was incised in the same manner. After incising the residual mucosa, a complete circumferential mucosal incision was made. Submucosal dissection was performed from the frontal area to the dis- 
tal area and from the edge to the center area without using the tunneling method. Finally, the lesion was removed en bloc. During dissection, a local injection and a hemostatic procedure were performed, if necessary. An intralesional steroid injection of triamcinolone acetonide or oral steroids was administered according to the operator's judgment, and mainly for lesions with a circumferential mucosal defect of more than 9/12. Triamcinolone acetonide (Kenacort; Bristol-Myers Squibb Co., Tokyo, Japan) was diluted with saline, and $40 \mathrm{mg}$ to $80 \mathrm{mg}$ was injected into the submucosal layer just after ESD, according to the method reported by Hanaoka et al. [8]. Oral intake of prednisolone was started at $30 \mathrm{mg} /$ day and was tapered gradually for weeks according to the method of Yamaguchi et al. [9]. Steroids were administered according to the judgment of the endoscopist since April 2010. The resected specimen was cut into a 2-mm section and examined histologically.

\section{Evaluation}

We evaluated procedure time, en bloc resection rate, $\mathrm{R} 0$ resection rate, number of cases under general anesthesia, and adverse events (AEs) to assess the safety of ESD. We also evaluated local recurrence rate, cause of death, and overall survival rate as measures of the efficacy of ESD. Tumor size was defined as tumor length in the long axis. Specimen size was defined as resected specimen length in the long axis. Procedure time was defined from incision to removal of specimen. $\mathrm{R} 0$ resection was defined as en bloc resection with negative horizontal and vertical margins. AEs included perforation, post-ESD bleeding, and esophageal stricture. Esophageal stricture was defined as when the endoscope could not be passed through the treatment site and when the patient presented with a chief complaint of dysphagia to semi-solid foods.

\section{Follow-up}

In patients with potential lymph node metastasis based on histological assessment, additional treatment was recommended. When patients were confirmed as having curative resection, they underwent upper gastrointestinal endoscopy every 6 to 12 months for follow-up after ESD. Curative resection was defined as a tumor that had been resected completely within a tumor-invasion depth to Lamina propuria muscle (LPM) with no lymphovascular involvement.

Long-term outcomes were assessed on an individual basis.

\section{Statistical analysis}

Continuous variables were compared using the Mann-Whitney test. Categorical variables were compared using the chisquared test or Fisher's exact test when appropriate. KaplanMeier curves were constructed to analyze rates of survival. A log-rank test was used to evaluate the significance of differences between curves. For all analyses, a $P$ value $<5 \%$ was considered to be significant. All statistical analyses were performed using STATA software (ver. 11, StataCorp LP, Texas, USA). Our study was approved by the institutional review board, and all study participants provided informed consent.

\section{Results}

Clinicopathological characteristics are shown in $>$ Table 1. Gender and mean age were similar in both groups, but mean tumor size was $20.7 \mathrm{~mm}$ in the case group and $24.2 \mathrm{~mm}$ in the control group. The rate of mucosal cancer in the case group was significantly higher than that in the control group. Frequency of having a history of treatment for esophageal cancer was $66.7 \%$ in the case group and $13.9 \%$ in the control group; this was a significant difference between the 2 groups.

Results of ESD are shown in > Table 2. En bloc resection was $100 \%$ in both groups. Mean procedure time was similar in both groups, but the number of ESDs performed under general anesthesia in the case group was higher than in the control group. The rate of AEs except esophageal stricture was small; perforation occurred in 4 cases. Esophageal stricture developed in $20 \%$ of the case group and in $6.6 \%$ of the control group. There was a significant difference between the 2 groups in this regard, although the degree of mucosal defect was not significant. Six patients in the case group received an intralesional steroid injection; 5 of these patients had a circumferential mucosal defect of more than $3 / 4$, and 1 had a defect of 2/3. Twenty-two patients in the control group received an intralesional steroid injection; 17 of them had a mucosal defect of more than 3/4, 4 had a defect of $2 / 3$, and 1 had a defect of $7 / 12$. Further, oral steroids were administered in 1 patient in the case group, in the patient with the mucosal defect of $11 / 12$, and in 2 patients in the control group, in whom the defect was more than 3/4. The patients who developed esophageal stricture in the case group included 4 of 6 cases with intralesional steroid injection, 1 case with oral steroid administration, and 4 cases without steroid administration. A subgroup analysis of the relationship between the preventative methods used and development of esophageal stricture according to extent of mucosal defect is shown in $>$ Table 3 . The analysis showed that esophageal stricture developed significantly more often in the case group than in the control group even though a steroid was injected into the artificial ulcer site when a circumferential mucosal defect of more than 3/4. In addition, the mean number of balloon dilatations performed was $21.1 \pm 13.1$ times. This was significantly higher than in the control group. No patients were referred for additional surgery in the case group, but 19 in the control group underwent further surgery based on their histology results. However, there was no significant difference in the referral rate between the case group and the control group. There was no local recurrence and no death from primary cancer and treatment-related disease in the case group. The 3-year survival rate was $88.4 \%$ in the case group and $96.7 \%$ in the control group, and there was no significant difference between them ( Fig.2).

\section{Discussion}

Cervical esophageal cancer is not common, and its biological and clinical features are seldom reported. According to Saeki $\mathrm{H}$ [10], female patients more frequently have this type of cancer, and the malignant potential of CEC is not higher than those for 
- Table1 Clinicopathological characteristics of study participants.

\begin{tabular}{|c|c|c|c|}
\hline & Case & Control & $P$ value \\
\hline Patients (lesions), n & $45(45)$ & $375(405)$ & \\
\hline Age, years (mean and SD) & $67.6 \pm 7.9$ & $67.3 \pm 9.2$ & 0.82 \\
\hline Sex & & & 0.48 \\
\hline - Male & 38 & 330 & \\
\hline - Female & 7 & 45 & \\
\hline Tumor size, mm (mean and SD) & $20.7 \pm 14.2$ & $24.2 \pm 14.9$ & 0.094 \\
\hline Mean specimen size, mm (mean and SD) & $32.3 \pm 14.5$ & $39.2 \pm 14.5$ & 0.0002 \\
\hline \multicolumn{4}{|l|}{ Paris classification } \\
\hline$=0-1$ & 0 & 10 & 0.34 \\
\hline - 0 -lla & 4 & 20 & 0.21 \\
\hline . $0-I I b$ & 22 & 153 & 0.10 \\
\hline - 0 -IIc & 19 & 222 & 0.074 \\
\hline Invasion & & & 0.04 \\
\hline . M & 44 & 354 & \\
\hline . SM & 1 & 51 & \\
\hline Previous history of esophageal cancer, n (\%) & $30(66.7)$ & $52(13.9)$ & $<0.0001$ \\
\hline Follow-up duration, months (median and range) & $37.7(0.3-84)$ & $49.7(0.2-106)$ & 0.044 \\
\hline
\end{tabular}

cancers of the thoracic and abdominal esophagus. However, these data were extracted from advanced cases treated by surgery. According to DG Grass [11], they collected 362 CEC patients from the Surveillance, Epidemiology, and End Results database of the United States. In patients with documented stage CEC, the majority of patients presented with locally advanced disease, with $50 \%$ being stage III, but $25 \%$ stage 1 . The majority of patients presented with locally advanced primary tumors, with $58 \%$ having a primary tumor classification of T3 or T4, and $34 \%$ having T1. The median overall survival and diseasespecific survival for the population were 14 and 16 months, respectively. Unfortunately, there has been no report on ESD for early-stage CEC. In this study, rates of mucosal cancer and history of esophageal cancer in the case group were higher than in the control group. This is one of the specific features of CEC that is expected to be a good indication for ESD. According to the Comprehensive Registry of Esophageal Cancer in Japan [12], these cancers are located mainly in the mid-thoracic esophagus and the number of cases of CEC is small. However, most patients are scheduled for follow-up endoscopy after esophagectomy so that early-stage CEC can be detected and treated endoscopically. Accordingly, there was a relatively increased rate of mucosal cancer and a greater number of patients with a previous history of esophageal cancer. To the best of our knowledge, this is the first study to demonstrate medium and shortterm clinical outcomes of ESD for CEC at an early stage.
The cervical esophagus is affected by the upper sphincter muscle, making it difficult not only to detect the lesions but also to treat them by ESD. We generally performed ESD under general anesthesia, so the lumen of cervical esophagus was easily spread due to muscle relaxant, and those conditions help to keep position and aid maneuverability during ESD. In addition, the tumor was located at the upper esophageal sphincter in 7 patients. This location is technically more challenging, but we successfully achieved en bloc resection by using direct or curved laryngoscopy, which helped to create a wider working space. As we have previously reported [13], this method is commonly used when performing ESD for pharyngeal cancer. Consequently, the en bloc resection rate was $100 \%$, and the R0 resection rate was $91.1 \%$. Moreover, neither perforation nor post ESD bleeding was observed in the case group. However, the incidence rate of esophageal stricture after ESD in the case group was significantly higher than those in the control group. Although the degree of mucosal defect and frequency of steroid administration did not differ between the cervical cases and middle thoracic cases, esophageal stricture developed in the cervical esophagus. The cause of the higher incidence of stricture in CEC is of great concern and there is no clear explanation for this as yet. We hypothesize that the cervical esophagus is usually contracted because of the upper sphincter musculature, and when the artificial ulcer starts to heal, not only fibrosis but also muscle contraction might contribute to the development of stricture. According to our data, 
- Table 2 Results of ESD for cervical esophageal cancer.

\begin{tabular}{|c|c|c|c|c|}
\hline & & Case & Control & $P$ \\
\hline \multicolumn{2}{|l|}{ Patients (lesions), $\mathrm{n}$} & $45(45)$ & $375(405)$ & \\
\hline \multicolumn{2}{|l|}{ En bloc resection rate (\%) } & 100 & 100 & - \\
\hline \multicolumn{2}{|l|}{ R0 resection rate (\%) } & 91.1 & 96 & 0.052 \\
\hline \multicolumn{2}{|l|}{ Mean procedure time (min) } & $57.0 \pm 49.6$ & $54.0 \pm 32.4$ & 0.36 \\
\hline \multicolumn{2}{|l|}{ Case under general anesthesia (\%) } & 71.1 & 11.1 & $<0.001$ \\
\hline \multicolumn{2}{|l|}{ Muscle injury during ESD (\%) } & 11.1 & 22.5 & 0.052 \\
\hline \multicolumn{5}{|l|}{ Adverse events } \\
\hline & Post ESD bleeding & 0 & 0 & - \\
\hline & Perforation & 1 & 3 & 0.35 \\
\hline & Delayed perforation & 0 & 0 & - \\
\hline & Post ESD stricture & $9(20 \%)$ & $14(6.6)$ & $<0.001$ \\
\hline \multirow[t]{2}{*}{ Mucosal defect } & Less than $1 / 2$ & 22 & 183 & 0.64 \\
\hline & More than $1 / 2$ & 23 & 222 & \\
\hline \multicolumn{2}{|l|}{ Intralesional steroid injection } & 6 & 22 & 0.049 \\
\hline \multicolumn{5}{|l|}{ The number of balloon dilatation } \\
\hline \multicolumn{2}{|l|}{ Mean median } & $21.1 \pm 13.1,22$ & $7.1 \pm 4.8,7$ & 0.0041 \\
\hline \multicolumn{2}{|l|}{ Oral administration } & 1 & 2 & 0.27 \\
\hline \multicolumn{2}{|l|}{ Additional treatment } & 1 & 68 & 0.004 \\
\hline & Surgery & 0 & 19 & 0.13 \\
\hline & CRT & 1 & 49 & 0.027 \\
\hline \multicolumn{2}{|l|}{ Local recurrence } & 0 & 1 & 0.89 \\
\hline \multirow[t]{3}{*}{ Cause of death } & Primary cancer & 0 & 5 & 0.57 \\
\hline & Other causes & 7 & 9 & 0.001 \\
\hline & Treatment-related disease & 0 & 0 & - \\
\hline
\end{tabular}

a single intralesional steroid injection was not enough to prevent esophageal stricture, especially when the circumferential mucosal defect was more than three quarters. Although our patient population was small, frequency of development of esophageal stricture was significantly higher in the case group than in the control group ( $80 \%$ vs $24 \%$ ). However, these data have a major limitation in that the decision to inject steroids was made by the operator, which contributed to there being no choice of a preventative measure in $45 \%$ of the case group and $78 \%$ of the control group, even though more than $75 \%$ of the circumferential mucosa was resected. We acknowledge that this was a source of bias in our study. Moreover, when stricture developed, a repeated balloon dilations were required and the number was significantly higher than for the middle thoracic esophagus. Our data raised concern as to whether esophageal stricture would lead to a high incidence of fatal aspiration pneumonia. However, analysis of the relationship between esophageal stricture and cause of death showed that 6 of 7 patients who died from other causes had been free from stricture until death. One patient who had a history of treatment for esophageal stricture died of leukemia and did not experience aspiration. Fortunately, in this study there was no evidence of a relationship between esophageal stricture and death.

Outcomes of ESD for superficial CEC were statistically noninferior to those for mid-thoracic esophageal cancers, even though three-quarters of the patients with CEC had a history of esophageal cancer. Our data show an R0 resection rate of more than $90 \%$, no local recurrence, and no deaths from primary CEC. Therefore, if the cancer can be detected at an early stage, endoscopic treatment may be effective not only for local control but potentially also for disease control. Further, the finding of no perforation or post-ESD bleeding in the case group indicates that ESD can be performed safely in patients with CEC. To find early cancer, the target should be examined carefully and focused on. Patients with superficial cancer of the cervical esophagus more frequently have a history of treatment of prior esophageal cancer. When we examined these patients, the cervical esophagus was the area of most concern. 
- Table 3 Subgroup analysis of the relationship between steroid administration and frequency of esophageal stricture according to the extent of mucosal defect.

\begin{tabular}{|l|l|l|l|}
\hline $1 / 2 \leqq$ mucosal defect<3/4 & \multicolumn{2}{|l|}{$\mathrm{n=146}$} & P value \\
\hline Positive for esophageal stricture & Case & Control & \\
\hline Intralesional steroids & $0 / 1$ & $1 / 5$ & 0.83 \\
\hline Oral steroids & $0 / 0$ & $0 / 0$ & 1.00 \\
\hline No treatment & $1 / 11$ & $0 / 129$ & 0.079 \\
\hline & & & \\
\hline $3 / 4 \leqq$ mucosal defect<1 & & & $P$ value \\
\hline Positive for esophageal stricture & Case & Control & \\
\hline Intralesional steroids & $4 / 5$ & $4 / 17$ & 0.039 \\
\hline Oral steroids & $1 / 1$ & $0 / 2$ & 0.33 \\
\hline No treatment & $3 / 5$ & $9 / 68$ & 0.029 \\
\hline
\end{tabular}

After examination, if early CEC had been detected, we considered carefully whether that lesion was an indication for ESD. Two patients in the case group died from progression or metastasis of primary esophageal cancer. The overall survival curve shown in > Fig. 2 included those cases. Although some selection bias may have been introduced at the time a decision was made about treatment with ESD, we believe that this approach clearly demonstrated the specific features of early-stage CEC.

There are some limitations of this study. First, it was retrospective, had a small sample size and was conducted at a single center. However, because the number of superficial CECs was not large, a randomized controlled study is impractical. To confirm our data, it would be better to collect data from a number of centers and analyze them. Second, there was a degree of selection bias in this study arising from exclusion of lesions with complete circumferential extension, recurrent lesions, and lesions with scarring in both study groups. Such cases are rare and technically challenging, and their data would not be representative of cases commonly encountered in clinical practice.

\section{Conclusion}

In conclusion, ESD was achieved safely for superficial CEC and successful local control was confirmed. Further, short-term and mid-term outcomes of ESD for early-stage CEC were statistically non-inferior to those for mid-thoracic esophageal tumors. However, esophageal stricture after ESD was more frequent. This is a problem that needs to be overcome in the future.

\section{Competing interests}

None

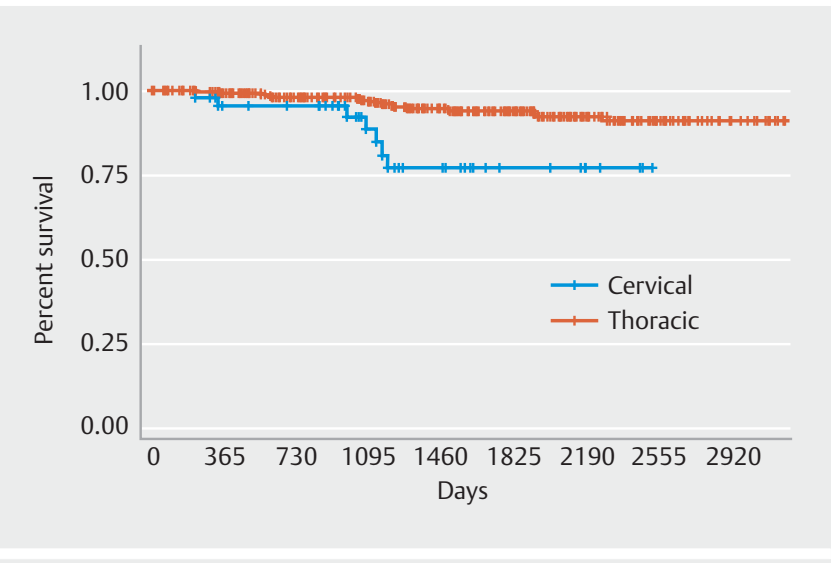

- Fig. 2 Overall survival curve in the case group and control group.

\section{References}

[1] Lee D], Harris A, Gillette A et al. Carcinoma of the cervical esophagus: diagnosis, management, and results. South Med J 1984; 77: 1365 1367

[2] Grass GD, Cooper SL, Armeson K et al. Cervical esophageal cancer: a population-based study. Head Neck 2015; 37: $808-814$

[3] Mendenhall WM, Sombeck MD, Parsons JT et al. Management of cervical esophageal carcinoma. Semin Radiat Oncol 1994; 4: 179-191

[4] Tepper J, Krasna MJ, Niedzwiecki D et al. Phase III trial of trimodality therapy with cisplatin, fluorouracil, radiotherapy, and surgery compared with surgery alone for esophageal cancer: CALGB 9781. J Clin Oncol 2008; 26: 1086 - 1092

[5] Daiko H, Hayashi R, Saikawa M et al. Surgical management of carcinoma of the cervical esophagus. J Surg Oncol 2007; 96: 166-172

[6] Shuangba H, Jingwu S, Yinfeng W et al. Complication following gastric pull-up reconstruction for advanced hypopharyngeal or cervical esophageal carcinoma: a 20-year review in a Chinese institute. Am J Otolaryngol 2011; 32: 275-278

[7] Hoebena A, Polak J, Van De Voorde L. Cervical esophageal cancer: a gap in cancer knowledge. Ann Oncol 2016; 27: 1664-1674

[8] Hanaoka N, Ishihara R, Takeuchi Y et al. Intralesional steroid injection to prevent stricture after endoscopic submucosal dissection for esophageal cancer: a controlled prospective study. Endoscopy 2012; 44: 1007-1011

[9] Yamaguchi N, Isomoto H, Nakayama T et al. Usefulness of oral prednisolone in the treatment of esophageal stricture after endoscopic submucosal dissection for superficial esophageal squamous cell carcinoma. Gastrointest Endosc 2011; 73: 1115-1121

[10] Saeki H, Tsutsumi S, Yukaya T et al. Clinicopathological Features of Cervical Esophageal Cancer: Retrospective Analysis of 63 Consecutive Patients Who Underwent Surgical Resection. Ann Surg 2017; 265: $130-136$

[11] Grass GD, Cooper SL, Armeson K et al. Cervical esophageal cancer: a population-based study. Head Neck 2015; 37: 808-814

[12] Tachimori Y, Ozawa S, Numasaki H et al. Comprehensive Registry of Esophageal Cancer in Japan, 2009. Esophagus 2016; 13: 110-137

[13] lizuka T, Kikuchi D, Hoteya S et al. Endoscopic submucosal dissection for treatment of mesopharyngeal and hypopharyngeal carcinomas. Endoscopy 2009; 41: 113-117 